\title{
Treatment of Congenital Microgastria
}

\author{
Larissa I. A. Ruczynski ${ }^{10}$ \\ Sanne M. B. I. Botden² \\ Ivo de Blaauw ${ }^{2}$ \\ ${ }^{1}$ Department of Research on Learning and Education, Radboudumc
Health Academy-Radboudumc, Nijmegen, The Netherlands
${ }^{2}$ Department of Pediatric Surgery, Radboudumc Amalia Children's
Hospital, Nijmegen, The Netherlands \\ Eur J Pediatr Surg 2021;31:129-134.
}

Horst E. Daniels-Scharbatke ${ }^{2}$

Maarten Schurink ${ }^{2}$

\author{
Address for correspondence Ivo de Blaauw, Department of Surgery/ \\ Pediatric Surgery, Radboudumc Amalia Children's Hospital, Geert \\ Grooteplein 10, 6525 GA Nijmegen, The Netherlands \\ (e-mail: Ivo.deBlaauw@radboudumc.nl).
}

\begin{abstract}
Keywords

- microgastria

- congenital microgastria

- Hunt-Lawrence pouch

- total esophageal gastric dissociation

- intestinal malrotation

Introduction Congenital microgastria is an extremely rare birth defect. The aim of this study was to present an overview of existing literature on the treatment of microgastria.

Materials and Methods The term "microgastria" was used in a PubMed and Medline search. Since merely case reports were found, only a narrative synthesis with limited statistical analysis can be given. Data of different treatment modalities were collected and divided into two groups: conservative or less invasive treatment (C/LT, i.e., modified diet or a gastrostomy/jejunostomy) and extensive gastric surgery (EGS, i.e., Hunt-Lawrence pouch or total esophageal gastric dissociation). Clinical outcome parameters (nutrition, growth pattern, and mortality) were compared.

Results Out of 73 articles published from 1973 to 2019, 38 articles describing 51 cases were included. In four patients, microgastria was an isolated anomaly (8\%). Type of treatment was described in only 46 patients, 19 were treated by C/LT. Mortality was $9 / 19(47 \%)$ in the C/LT group versus $4 / 27(15 \%)$ in the EGS group (chi-square $=5.829$, $p=0.016$, Fisher $=0.022$ ). There was a negative correlation between the invasiveness of the treatment and both mortality $(r=-0.356, p=0.015)$ and comorbidity $(r=-0.506, p<0.001)$. Patients in the C/LT group had significantly more comorbidity than in the EGS group (mean $=4.32$ vs. 2.26, $p=0.001$ ). There was a positive correlation between comorbidity and mortality $(r=0.400, p=0.006)$. Median follow-up was 42 months (range: 1-240). Type and way of nutrition were poorly described. In at least 9 of the 33 surviving patients, oral feeding was reported as normal, of whom 8 belonged to the EGS group. In all patients, growth could be acknowledged, but in comparison to peers, final body length was less. There was no difference in final body length between the two treatment groups.

Conclusion In patients with congenital microgastria, only minimal differences in clinical outcome in terms of type of nutrition and body growth were found when C/LT was compared with treatment by EGS. Mortality was significantly higher in the first group as well as the amount of comorbidities.
\end{abstract}

received

September 6, 2019

accepted

March 27, 2020

published online

May 18, 2020 (c) 2020. Thieme. All rights reserved.

Georg Thieme Verlag KG,

Rüdigerstraße 14,

70469 Stuttgart, Germany
DOI https://doi.org/

10.1055/s-0040-1710027.

ISSN 0939-7248. 


\section{Introduction}

Throughout the literature, less than 100 cases of congenital microgastria have been described. ${ }^{1}$ Microgastria is a rare condition and often associated with other anomalies, such as asplenia, intestinal malrotation, diaphragmatic hernia, cardiopulmonary anomalies, renal anomalies, limb defects, and laryngotracheobronchial clefts. ${ }^{2-36}$ Patients can show symptoms of food intolerance, gastroesophageal reflux, vomiting, recurrent aspiration pneumonia, and failure to thrive. Several treatment options have been described, but since 1980, various more extensive surgical procedures are being performed. ${ }^{4}$ However, long-term results of these surgical interventions remain unclear. In this study, we present an overview of existing literature on the outcome of both more extensive surgical and minimal invasive or nonsurgical treatment options.

\section{Materials and Methods}

A literature search was conducted. Congenital microgastria is not a known MeSH term; therefore, a PubMed and Medline search was performed using the full-text term "microgastria." Study relevance was evaluated by screening title and abstract. Articles not written in English or without online availability were excluded, as well as articles on microgastria not describing its treatment. A graphic resume is presented as a flowchart in -Fig. 1. After the final selection of articles was determined, all data were pooled according to the type of treatment. Due to the fact that only case reports were being found and due to the small number of patients, PRISMA guidelines were found not suitable to analyze the results. ${ }^{37}$ Therefore, a narrative synthesis is given.

To compare the different treatment modalities, we divided them into two groups: conservative or less invasive treatment (C/LT, i.e., modified diet, gastrostomy or jejunostomy) and

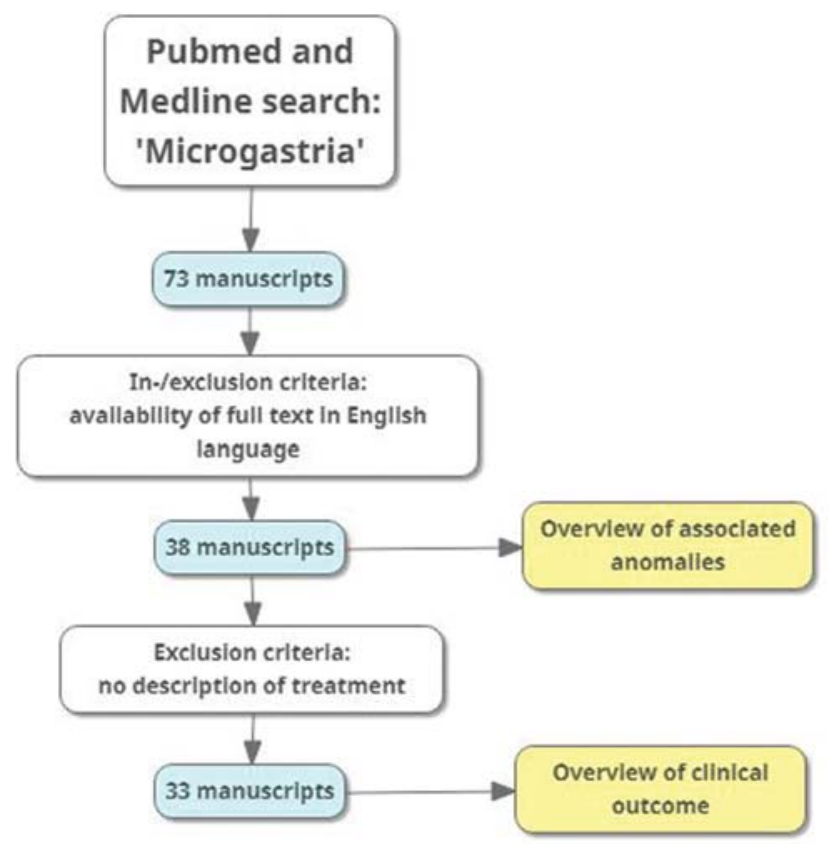

Fig. 1 Flowchart. treatment by extensive gastric surgery (EGS, i.e., Hunt-Lawrence pouch or total esophageal gastric dissociation [TEGD]). For statistical analysis, we compared the different groups using correlation tests, $t$-tests, chi-square tests, and Fisher's exact tests in SPSS version 25.

\section{Results}

Online literature search identified 71 articles published between 1973 and 2019. All articles not written in English or without online availability were excluded, after which an overview of associated anomalies, as presented in - Table 1, was composed. Noteworthy is the fact that in only four patients $(4 / 51 ; 8 \%)$, microgastria was present as an isolated anomaly. ${ }^{1,16,38,39}$ The most common associated anomalies are defects of the spleen and the limbs.

As 5 of the remaining 38 articles did not describe any form of treatment of congenital microgastria, $, 12,15,18,3133$ relevant articles remained for a narrative synthesis of clinical outcome and limited statistical synthesis. In total, 46 patients with congenital microgastria were found, of whom 19 were treated conservatively ( - Table 2). - Table 3 summarizes the clinical outcome in the two treatment groups. Mortality was 9/19(47\%) in the C/LT group versus 4/27 (15\%) in the EGS group which was confirmed as a significant difference (chi-square $=5.829$, $p=0.016$, Fisher $=0.022$ ). There was a negative correlation between the invasiveness of the treatment and both mortality $(r=-0.356, p=0.015)$ and comorbidity $(r=-0.506, p<0.001)$. Patients in the C/LT group had significantly more comorbidity than in the EGS group (mean $=4.32$ vs. 2.26, $p=0.001$ ). Comorbidities that were significantly associated with the invasiveness of the treatment were the categories "other intraabdominal anomalies" (chi-square $=5.906, p=0.015$, Fisher $=0.025$ ) and "central nervous system defects" (chisquare $=5.888, p=0.015$, Fisher $=0.032$ ). There was a positive correlation between comorbidity and mortality $(r=0.400$, $p=0.006$ ). The only significant association between a certain category of comorbidity and mortality was found for anorectal malformations (chi-square $=8.147, p=0.004$, Fisher $=0.019$ ).

Looking at the deceased patients, we found that they have significantly more comorbidity in the categories mentioned in -Table 1 than the group of surviving patients ( mean $=4.38$ vs. $2.61, p=0.032$ ). In the C/LT group, mortality was mostly related to comorbidity and not to the microgastria itself, whereas in the EGS group, the highest mortality rate was found in patients who underwent several other operations. ${ }^{26,27}$ The most frequently found EGS treatment was the Hunt-Lawrence pouch. In reports describing the reconstruction of a Hunt-Lawrence pouch, a relatively low mortality rate of $6 \%$ was found.

Of all surviving patients, outcomes of nutrition and growth are shown in - Table 3 . In general, the exact amount and type of feeding were poorly described. In the C/LT group, only $6 / 10$ could be enterally fed, either in frequent small amounts $(n=3)$, through enteral feedings $(n=2)$ or normally $(n=1) .^{22}$ In four patients, no details on enteral feeding were available. In the EGS group, data on enteral feeding were available in $18 / 23$ patients and consisted of a normal 
Table 1 Associated anomalies ranked by category

\begin{tabular}{|l|l|l|l|l|}
\hline Comorbidity & Total $(\% ; \boldsymbol{N}=\mathbf{5 1})$ & C/LT $(\% ; \boldsymbol{N = 1 9 )}$ & EGS (\%; $\boldsymbol{N = 2 7 )}$ & NUT (\%; $\boldsymbol{N = 5 )}$ \\
\hline Spleen anomalies & $20(39)$ & $7(37)$ & $9(33)$ & $4(80)$ \\
\hline Limb anomalies & $19(37)$ & $10(53)$ & $6(22)$ & $3(60)$ \\
\hline Urogenital malformations & $18(35)$ & $11(58)$ & $5(19)$ & $2(40)$ \\
\hline Facial dysmorphia & $16(31)$ & $6(32)$ & $7(26)$ & $3(60)$ \\
\hline Other intra-abdominal anomalies & $16(31)$ & $9(47)$ & $5(19)$ & $2(40)$ \\
\hline Cardiovascular anomalies & $15(29)$ & $6(32)$ & $8(30)$ & $1(20)$ \\
\hline Intestinal malrotation & $14(27)$ & $6(32)$ & $6(22)$ & $2(40)$ \\
\hline CNS anomalies & $10(20)$ & $7(37)$ & $2(7)$ & $1(20)$ \\
\hline Lung anomalies & $9(18)$ & $3(16)$ & $3(11)$ & $3(60)$ \\
\hline Esophageal atresia & $7(14)$ & $4(21)$ & $2(7)$ & $1(20)$ \\
\hline Congenital hearing loss & $6(12)$ & $3(16)$ & $3(11)$ & $0(0)$ \\
\hline Diaphragmatic hernia & $6(12)$ & $2(11)$ & $3(11)$ & $1(20)$ \\
\hline Vertebral anomalies & $6(12)$ & $4(21)$ & $2(7)$ & $0(0)$ \\
\hline Anorectal malformation & $4(8)$ & $3(16)$ & $0(0)$ & $1(20)$ \\
\hline No other anomalies & $4(8)$ & $0(0)$ & $4(15)$ & $0(0)$ \\
\hline Metabolic disorder & $1(2)$ & $1(5)$ & $0(0)$ & $0(0)$ \\
\hline
\end{tabular}

Abbreviations: C/LT, conservative or less invasive treatment; CNS, central nervous system; EGS, extensive gastric surgery; NUT, no or unknown treatment. Note: Of 51 reported cases, most patients have multiple comorbidity.

diet in 8 patients (35\%) and the need for additional nocturnal feedings in 4 patients (13\%). The patients in both groups seemed to grow adequately, although they remain relatively small compared with their peers when looking at the World Health Organization or national growth charts. Median follow-up was 42 months (range: $1-240$ ).

\section{Discussion}

Congenital microgastria is an early defect in the embryological development of the foregut and often associated with other anomalies, the most frequent being anomalies of the spleen and limbs. Both facial dysmorphisms and limb anomalies are often seen in syndromal disorders ${ }^{5-7,9,15-18,21,23,26,29,30,33-36}$; however, only a few syndromal cases have been reported in the literature, for example, the Pierre Robin sequence. ${ }^{23,30,33,35}$ The foregut starts to grow from the level of the pharynx, forming esophageal and gastric precursors as well as the lungs. In the fifth week, the stomach originates from the dorsal mesogastrium along with the spleen. This explains the association with lung anomalies, esophageal atresia, diaphragmatic hernia, and asplenia. Thus, the clinical manifestation can be determined by the moment in the embryological timeline where the defect finds its origin. In most cases of microgastria, the stomach is nothing more than a small saccular, or tubular, midsagittal structure with minimal reservoir capacity. If not diagnosed at an early age, a dilated esophagus can be found as a result of compensation for the small reservoir.

Histological analysis usually shows a normal cell differentiation with a lagging cell number. ${ }^{8}$ Depending on whether the cell differentiation was entirely completed or not, the stomach shows some functionality and produces a certain amount of acid and intrinsic factor. Therefore, the stomach appears to be both anatomically and functionally rudimentary at birth and it is hard to predict possible growth and functional outcome after birth.

In 1980, the first surgical treatment for microgastria was published, and before 1980 , many patients with microgastria died at an early age. ${ }^{4}$ Currently, several treatment options exist, varying from conservative (such as a modified nutrition with or without a nasoduodenal tube) or less invasive surgery (i.e., gastrostomy, jejunostomy) to EGS (i.e., a HuntLawrence pouch, TEGD, or a combination thereof). A specific treatment is best chosen depending on the comorbidity and the failure of gastric and/or enteral feeding. It has been recommended to consult a clinical geneticist in cases of microgastria with several other anomalies to rule out specific syndromes. Anomalies that are part of these syndromes could cause a higher chance of mortality, and in some patients, they were the direct cause of death and not the presence of the microgastria. ${ }^{8-11,21,26,29}$ The mortality rate of the C/LT group was significantly higher than in the EGS group. We suspect that this could be due to the fact that patients with this treatment had an a priori worse outcome assignable to their comorbidity and that no major surgery was considered. The fact that there was significantly more comorbidity in the C/LT group than in the EGS group, the positive correlation between comorbidity and mortality and the fact that the group of deceased patients had significantly more comorbidity support this hypothesis.

Neifeld et al were the first to report for a successful use of the Hunt-Lawrence pouch in 1980. It consists of a double-lumen jejunal pouch which is attached to the greater curvature of the stomach with a distal Roux-en-Y jejunojejunostomy. ${ }^{4}$ Since 
132 Treatment of Congenital Microgastria Ruczynski et al.

Table 2 Treatment overview and mortality rate

\begin{tabular}{|c|c|c|c|c|c|c|c|}
\hline \multirow[t]{2}{*}{ Year } & \multirow[t]{2}{*}{ Authors } & \multirow[t]{2}{*}{$N$} & \multicolumn{2}{|c|}{$\begin{array}{l}\text { Conservative or less invasive } \\
\text { treatment }\end{array}$} & \multicolumn{3}{|c|}{ Extensive gastric surgery } \\
\hline & & & Nonoperative & $\begin{array}{l}\text { Gastrostomy/ } \\
\text { Jejunostomy }\end{array}$ & Hunt-Lawrence & TEGD & Other \\
\hline 1973 & Blank and Chisolm & 1 & & 1 & & & \\
\hline 1974 & Hochberger and Swoboda & 1 & 1 & & & & \\
\hline 1980 & Neifeld et al & 1 & & & 1 & & \\
\hline 1983 & Anderson and Guzzetta & 2 & & & 2 & & \\
\hline 1987 & Dorney et al & 1 & & & $1(1)$ & & \\
\hline 1990 & Velasco et al & 4 & $1(1)$ & & 3 & & \\
\hline 1992 & Meinecke et al & 1 & $1(1)$ & & & & \\
\hline 1993 & Cunniff et al & 3 & $1(1)$ & & 2 & & \\
\hline 1993 & Hasegawa et al & 1 & & $1(1)$ & & & \\
\hline 1994 & Hoehner et al & 1 & & & 1 & & \\
\hline 1994 & Moulton et al & 1 & & & 1 & & \\
\hline 1996 & Ramos et al & 1 & & & 1 & & \\
\hline 1997 & Hernáiz Driever et al & 1 & 1 & & & & \\
\hline 1997 & Sarin et al & 1 & 1 & & & & \\
\hline 1998 & Kroes and Festen & 1 & & & 1 & & \\
\hline 1999 & al-Gazali et al & 2 & $1(1)$ & $1(1)$ & & & \\
\hline 1999 & Murray et al & 1 & & 1 & & & \\
\hline 2000 & Giurgea et al & 1 & & & & & $1^{\mathrm{a}}$ \\
\hline 2002 & Stewart et al & 1 & 1 & & & & \\
\hline 2003 & Menon et al & 1 & & & 1 & & \\
\hline 2004 & Herman and Siegel & 1 & & 1 & & & \\
\hline 2005 & Kawaguchi et al & 6 & & $2(2)$ & & $2(1)$ & $2(1)^{b}$ \\
\hline 2005 & Sharma and Menon & 1 & & & & & $1(1)^{c}$ \\
\hline 2006 & Lall et al & 1 & & & & 1 & \\
\hline 2007 & Jones and Cohen & 1 & & & 1 & & \\
\hline 2008 & Filippi et al & 1 & $1(1)$ & & & & \\
\hline 2008 & Laurie and Wakeling & 1 & & 1 & & & \\
\hline 2010 & Dicken et al & 2 & & & 2 & & \\
\hline 2011 & Vasas et al & 1 & & 1 & & & \\
\hline 2011 & Kunisaki et al & 1 & & & & 1 & \\
\hline 2014 & Roberts et al & 1 & & 1 & & & \\
\hline 2016 & Hattori et al & 1 & & & & 1 & \\
\hline 2017 & Filisetti et al & 1 & & & 1 & & \\
\hline \multirow{2}{*}{\multicolumn{3}{|c|}{ Mortality }} & $5 / 9(56 \%)$ & $4 / 10(40 \%)$ & $1 / 18(6 \%)$ & $1 / 5(20 \%)$ & $2 / 4(50 \%)$ \\
\hline & & & \multicolumn{2}{|l|}{$9 / 19(47 \%)$} & \multicolumn{3}{|l|}{$4 / 27(15 \%)$} \\
\hline
\end{tabular}

Abbreviation: TEGD, total esophageal gastric dissociation.

Note: Number presented within () denotes mortality rate.

${ }^{a}$ Duodenal diversion, fundojejunal anastomosis in a jejunal pouch + gastrostomy.

${ }^{b}$ Combination of stomach division, a fundoplication and a Roux-en-Y jejunostomy $(N=2)$.

'Diamond-shaped side-to-side anastomosis between distal esophagus and stomach (to bypass the stenosis of the gastroesophageal transition).

then, this procedure has been applied in roughly 18 cases with variable success. ${ }^{1,4,5,7,8,10,13,14,16,20,32,38,39}$ Kawaguchi et al introduced the use of the TEGD. ${ }^{26}$ Up until now, this procedure has been performed five times, with good outcome being reported on gastroesophageal reflux symptoms. ${ }^{26,28,33,36}$ Growth and nutrition have not been described properly in all cases but known data mentioned adequate growth and normal nutrition. 
Table 3 Treatment overview, mortality rate, and outcome

\begin{tabular}{|c|c|c|c|c|c|c|}
\hline & \multicolumn{2}{|l|}{ C/LT } & \multicolumn{3}{|l|}{ EGS } & \multirow[t]{2}{*}{ NUT } \\
\hline & Nonoperative & $\begin{array}{l}\text { Gastrostomy/ } \\
\text { jejunostomy }\end{array}$ & Hunt-Lawrence & TEGD & Other & \\
\hline No. of patients & 9 & 10 & 18 & 5 & 4 & 5 \\
\hline No. of mortality & 5 & 4 & 1 & 1 & 2 & 2 \\
\hline Percentage & 56 & 40 & 6 & 20 & 50 & 40 \\
\hline Total mortality & \multicolumn{2}{|l|}{$9 / 19(47 \%)$} & \multicolumn{3}{|l|}{$4 / 27(15 \%)$} & $2 / 5(40 \%)$ \\
\hline Nutrition $^{\mathrm{b}}$ & \multicolumn{2}{|c|}{$\begin{array}{l}\text { Normal diet }(1 / 10) \\
\text { Frequent small amounts }(3 / 10) \\
\text { Enteral feedings }(2 / 10) \\
\text { No information }(4 / 10)\end{array}$} & \multicolumn{3}{|c|}{$\begin{array}{l}\text { Normal diet }(9 / 23) \\
\text { Frequent small amounts }(1 / 23) \\
\text { Additional nocturnal feedings }(6 / 23) \\
\text { Nutrition via tube }(2 / 23) \\
\text { No information }(5 / 23)\end{array}$} & $\begin{array}{l}\text { Not applicable or } \\
\text { not described }\end{array}$ \\
\hline Median growth ${ }^{\mathrm{b}}$ & \multicolumn{2}{|c|}{$\begin{array}{l}\text { Length: p35 (range: p25-50) } \\
\text { Weight: p25 (range: p15-50) }\end{array}$} & \multicolumn{3}{|c|}{$\begin{array}{l}\text { Length: p10 (range: p3-50) } \\
\text { Weight: p11 (range: p3-90) }\end{array}$} & $\begin{array}{l}\text { Not applicable or } \\
\text { not described }\end{array}$ \\
\hline
\end{tabular}

Abbreviations: C/LT, conservative or less invasive treatment; EGS, extensive gastric surgery; NUT, no or unknown treatment; TEGD, total esophageal

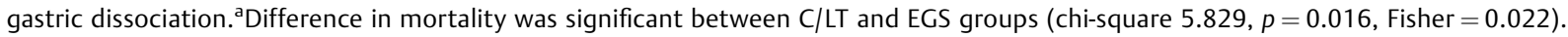

${ }^{\mathrm{b}}$ Of surviving patients.

Although the C/LT group had a higher mortality rate, the patients who survived had a good outcome on nutrition and growth (-Table 3 ). The EGS group had a lower mortality rate with similar growth and slightly better outcome of nutrition; however, case series are all small and the true benefit of major surgery in relation to possible enteral nutrition and growth remains to be determined. Extensive surgery comes with the burden of a large procedure in a vulnerable infant, generally with comorbidity of other organ structures as well. Overall, there is a relatively growth retardation observed in almost all patients with microgastria compared with their peers in the normal population. This seems to be independent of any surgical procedure.

Limitations of the study were the overall small number of patients and the fact that only case reports and case series have been published. Furthermore, these reports often showed incomplete data on nutrition and outcome of growth and development. Another important pitfall of this overview is the chance of publication bias. There are only a few cases seen in all surgical centers what comes with the possibility that the patients who are treated unsuccessfully are not published in the literature. Furthermore, there is also the probability that some cases of microgastria have been left unfound due to an early death of the patient. Then, the comparison of mortality between the different treatment groups should be interpreted carefully since the described follow-up time was varied. Finally, a fair comparison was hard to make because of the heterogeneity between both the treatment groups, considering the more complex comorbidity present in the C/LT group.

\section{Conclusion}

In patients with congenital microgastria, only minimal differences in clinical outcome in terms of type of nutrition and body growth were found when C/LT was compared with treatment by more EGS. Mortality was higher in the former group, but this may be related to severe comorbidities. To rule out possible associated syndromes, we recommend consultation of a clinical geneticist. Some form of registration in a database for rare anomalies seems desirable to improve the care of these patients. The recent start of European Reference Networks, including one for rare inherited and congenital anatomical anomalies (ERNICA: European Reference Network on rare Inherited and Congenital Anomalies), may be the first step to accomplish this. ${ }^{40}$ ERNICA: European Reference Network on Rare inherited and congenital anomalies

Based on these findings, it seems appropriate to adhere to a tailored treatment strategy and to only consider EGS when conservative or less invasive options have been deemed unsuccessful.

Conflict of Interest

None declared.

\section{References}

1 Jones VS, Cohen RC. An eighteen year follow-up after surgery for congenital microgastria-case report and review of literature.J Pediatr Surg 2007;42(11):1957-1960

2 Blank E, Chisolm AJ. Congenital microgastria, a case report with a 26-year follow-up. Pediatrics 1973;51(06):1037-1041

3 Hochberger O, Swoboda W. Congenital microgastria. A follow-up observation over six years. Pediatr Radiol 1974;2(03):207-208

4 Neifeld JP, Berman WF, Lawrence W Jr, Kodroff MB, Salzberg AM. Management of congenital microgastria with a jejunal reservoir pouch. J Pediatr Surg 1980;15(06):882-885

5 Anderson KD, Guzzetta PC. Treatment of congenital microgastria and dumping syndrome. J Pediatr Surg 1983;18(06):747-750

6 Mandell GA, Heyman S, Alavi A, Ziegler MM. A case of microgastria in association with splenic-gonadal fusion. Pediatr Radiol 1983;13(02):95-98

7 Dorney SF, Middleton AW, Kozlowski K, Benjamin BN, Kan AE, Kamath KR. Congenital agastria. J Pediatr Gastroenterol Nutr 1987;6(02):307-310

8 Velasco AL, Holcomb GW III, Templeton JM Jr, Ziegler MM. Management of congenital microgastria. J Pediatr Surg 1990;25 (02):192-197 
9 Meinecke P, Bönnemann CG, Laas R. Microgastria-hypoplastic upper limb association: a severe expression including microphthalmia, single nostril and arhinencephaly. Clin Dysmorphol 1992;1(01):43-46

10 Cunniff C, Williamson-Kruse L, Olney AH. Congenital microgastria and limb reduction defects. Pediatrics 1993;91(06):1192-1194

11 Hasegawa S, Kohno S, Tamura K, Urushihara N. Congenital microgastria in an infant with the VACTERL association. J Pediatr Surg 1993;28(06):782-784

12 Hill LM. Congenital microgastria: absence of the fetal stomach and normal third trimester amniotic fluid volume. J Ultrasound Med 1994;13(11):894-896

13 Hoehner JC, Kimura K, Soper RT. Congenital microgastria. J Pediatr Surg 1994;29(12):1591-1593

14 Moulton SL, Bouvet M, Lynch FP. Congenital microgastria in a premature infant. J Pediatr Surg 1994;29(12):1594-1595

15 Lurie IW, Magee CA, Sun CC, Ferencz C. 'Microgastria-limb reduction' complex with congenital heart disease and twinning. Clin Dysmorphol 1995;4(02):150-155

16 Ramos CT, Moss RL, Musemeche CA. Microgastria as an isolated anomaly. J Pediatr Surg 1996;31(10):1445-1447

17 Hernáiz Driever P, Göhlich-Ratmann G, König R, et al. Congenital microgastria, growth hormone deficiency and diabetes insipidus. Eur J Pediatr 1997;156(01):37-40

18 Moore PJ, Hawkins EP, Galliani CA, Guerry-Force ML. Splenogonadal fusion with limb deficiency and micrognathia. South Med J 1997;90(11):1152-1155

19 Sarin YK, Stephen E. Congenital microgastria. Indian Pediatr 1997; 34(02):157-158

20 Kroes EJ, Festen C. Congenital microgastria: a case report and review of literature. Pediatr Surg Int 1998;13(5-6):416-418

21 al-Gazali LI, Bakir M, Dawodu A, Nath R, al-Tatari HM, Gerami M. Recurrence of the severe form of microgastria-limb reduction defect in a consanguineous family. Clin Dysmorphol 1999;8(04): 253-258

22 Murray KF, Lillehei CW, Duggan C. Congenital microgastria: treatment with transient jejunal feedings. J Pediatr Gastroenterol Nutr 1999;28(03):343-345

23 Giurgea I, Raqbi F, Nihoul-Fékété C, Couly G, Abadie V. Congenital microgastria with Pierre Robin sequence and partial trismus. Clin Dysmorphol 2000;9(04):307-308

24 Stewart C, Stewart M, Stewart F. Microgastria-limb reduction anomaly with total amelia. Clin Dysmorphol 2002;11(03):187-190

25 Herman TE, Siegel MJ. Imaging casebook. Asplenia syndrome with congenital microgastria and malrotation. J Perinatol 2004;24(01): $50-52$
26 Kawaguchi AL, Donahoe PK, Ryan DP. Management and long-term follow-up of patients with types III and IV laryngotracheoesophageal clefts. J Pediatr Surg 2005;40(01):158-164

27 Sharma SC, Menon P. Congenital microgastria with esophageal stenosis and diaphragmatic hernia. Pediatr Surg Int 2005;21(04): 292-294

28 Lall A, Morabito A, Bianchi A. "Total Gastric Dissociation (TGD)" in difficult clinical situations. Eur J Pediatr Surg 2006;16(06):396-398

29 Filippi L, Serafini L, Fiorini P, Agostini E, Giovannucci Uzielli ML. Congenital microgastria and primary ciliary dyskinesia in a newborn with DiGeorge syndrome and 22q11.2 deletion. Eur J Pediatr Surg 2008;18(03):195-197

30 Laurie DE, Wakeling EL. Congenital microgastria in association with Pierre-Robin sequence. Clin Dysmorphol 2008;17(02):143-144

31 Nagendran S, Johal N, Set P, Brain J, Aslam A, Samuel M. Bilateral communicating intralobar sequestration and microgastria. Ann Thorac Surg 2009;88(06):2040

32 Dicken BJ, Novotny NM, Breckler FD, Yim JP, Rescorla FJ. Use of the Hunt-Lawrence pouch in congenital microgastria-a report of 2 cases. J Pediatr Surg 2010;45(11):2238-2240

33 Kunisaki SM, Dakhoub A, Jarboe MD, Geiger JD. Gastric dissociation for the treatment of congenital microgastria with paraesophageal hiatal hernia. J Pediatr Surg 2011;46(06):e1-e4

34 Vasas P, Mudan SS, Akle CA. Congenital microgastria with limb defect combined with megaduodenum: case report and review of literature. Indian J Surg 2011;73(02):122-124

35 Roberts J, Torres-Martinez W, Farrow E, et al. A case of Robin sequence, microgastria, radiohumeral synostosis, femoral deficiency, and other unusual findings: a newly recognized syndrome? Am J Med Genet A 2014;164A(02):287-290

36 Hattori K, Bvulani B, Numanoglu A, Cox SG, Millar AJ. Total esophageal gastric dissociation for the failed antireflux procedure in a child with microgastria. European J Pediatr Surg Rep 2016;4(01):6-9

37 Moher D, Liberati A, Tetzlaff J, Altman DG; PRISMA Group. Preferred reporting items for systematic reviews and meta-analyses: the PRISMA statement. BMJ 2009;339:b2535

38 Menon P, Rao KL, Cutinha HP, Thapa BR, Nagi B. Gastric augmentation in isolated congenital microgastria. J Pediatr Surg 2003;38 (10):E4-E6

39 Filisetti C, Maestri L, Meroni M, Marinoni F, Riccipetitoni G. Severe dumping syndrome in a 6-year-old girl with congenital microgastria treated by Hunt-Lawrence pouch. European J Pediatr Surg Rep 2017;5(01):e17-e20

40 ERNICA: European Reference Network on rare Inherited and Congenital Anomalies. Available at: https://www.orpha.net/consor/cgibin/OC_Exp.php?lng=NL\&Expert=483645. Accessed April 11, 2020 